\title{
Research on the Stochastic Hopf Bifurcation of the Shallow Lake Ecosystem with Stochastic Excitation
}

\author{
Ruichao $\mathrm{Li}^{1}$, Bo Wang ${ }^{1,2^{*}}$ \\ ${ }^{1}$ School of Mathematics and Statistics, Henan University, 475004, Kaifeng, Henan, China \\ ${ }^{2}$ Institute of Applied Mathematics, Henan University, 475004,Kaifeng, Henan, China \\ *15993353268@163.com
}

Keywords: Stochastic averaging method. The multiplicative stochastic excitation. Stochastic Hopf bifurcation. Regime shift.

Abstract: Establishing the model of the shallow lake ecosystem with stochastic excitation, with the stochastic averaging method and nonlinear dynamic theory, the model of the shallow lake ecosystem with stochastic excitation was simplified. Based on the Stratonovich-Khasminiskii stochastic average principle and Oseledec multiplicative ergodic theory. We studied the stochastic Hopf bifurcation behavior with the FPK method. The results showed that the bifurcation behavior of the stochastic system is different from the bifurcation behavior of the deterministic system. The effect of stochastic factors can make the stability of the system appear change. A pseudo-random noise can make the system appear regime shift. The bifurcation value of the system shifts.

\section{Introduction}

The shallow lake ecosystem exists alternative stable states. The transitions between the different stable states can occur with certain conditions. The change of system parameters can lead to the transitions between the different stable states [1,2]. Likewise, the effect of stochastic factors can also lead to regime shift. Considering a two-variable shallow lake ecosystem model, which is developed by Scheffer et al [3]. Considering the uncertainty of the initial field and parameter of model, Wang Bo and Qi Qianqian have studied by the CNOP method [4].

However, the shallow lake ecosystem is interfered by various stochastic factors in the real environment. For example, the wind and waves, air pressure, the rise and fall with water level, human interference and so on. For more accurately describing the phenomenon of transitions between the different stable states. Adding the stochastic item is essential and reasonable in the deterministic model.

\section{The Stochastic Model}

Establishing Stochastic Model. Considering the two-variable model of Scheffer et al, which describes the relation between macrophytes covers $V$ and turbidity $E$. The model is as follows:

$$
\left\{\begin{array}{l}
\frac{\mathrm{d} E}{\mathrm{~d} t}=r_{E} E\left(1.0-\frac{E}{E_{0}} \frac{h_{V}+V}{h_{V}}\right), \\
\frac{\mathrm{d} V}{\mathrm{~d} t}=r_{V} V\left(1.0-V \frac{h_{E}^{4}+E^{4}}{h_{E}^{4}}\right) .
\end{array}\right.
$$

Where $E$ means the vertical light attenuation in the shallow lake ecosystem, which represents the turbidity in water. $V$ means the macrophytes covers. $E_{0}$ is the control parameter, which represents the vertical light attenuation without macrophytes. In this model, the detail introduction is given in reference [3]. 
In this paper, we only considered the multiplicative stochastic excitation which is related to environment and its internal factor. The stochastic model is as follows:

$$
\left\{\begin{array}{l}
\frac{\mathrm{d} E}{\mathrm{~d} t}=r_{E} E\left(1.0-\frac{E}{E_{0}} \frac{h_{V}+V}{h_{V}}\right)+\sigma_{1} E \propto \xi(t), \\
\frac{\mathrm{d} V}{\mathrm{~d} t}=r_{V} V\left(1.0-V \frac{h_{E}^{4}+E^{4}}{h_{E}^{4}}\right)+\sigma_{2} V \text { o }(t) .
\end{array}\right.
$$

This model is stochastic differential equations with Stratonovich means. $\sigma_{1}$ and $\sigma_{2}$ are noise intensity. For ease of comparison studies, we suppose that the stochastic excitation $\xi(t)$ and $\eta(t)$ are independent Gaussian white noise with zero mean and standard deviation.

The Stochastic Averaging Method. For establishing stochastic model (2), we use the stochastic averaging method [5,6]. At first, by means of variable substitution: $y_{1}=E-E^{*}, y_{2}=V-V^{*},\left(E^{*}, \mathrm{~V}^{*}\right)$ is the equilibrium point of the deterministic model (1).

$$
\left\{\begin{array}{l}
\frac{\mathrm{d} y_{1}}{\mathrm{~d} t}=r_{E}\left(y_{1}+E^{*}\right)\left(1.0-\frac{\left(y_{1}+E^{*}\right)}{E_{0}} \frac{\left(h_{V}+y_{2}+V^{*}\right)}{h_{V}}\right)+\sigma_{1}\left(y_{1}+E^{*}\right) \propto \xi(t), \\
\frac{\mathrm{d} y_{2}}{\mathrm{~d} t}=r_{V}\left(y_{2}+V^{*}\right)\left(1.0-\left(y_{2}+V^{*}\right) \frac{h_{E}^{4}+\left(y_{1}+E^{*}\right)^{4}}{h_{E}^{4}}\right)+\sigma_{2}\left(y_{2}+V^{*}\right) \text { o }(t) .
\end{array}\right.
$$

The stochastic bifurcation of the equilibrium point $\left(E^{*}, \mathrm{~V}^{*}\right)$ of the stochastic model (2) translates into the stochastic bifurcation of the equilibrium point $(0,0)$ of the stochastic model (3). For the stochastic model (3), by means of variable substitution: $y_{1}=a \cos \theta, y_{2}=a \sin \theta$. Using the stochastic averaging method, the Ito stochastic differential equation is as follows:

$$
\left\{\begin{array}{l}
\mathrm{d} a=m_{a} \mathrm{dt}+\sigma_{11} \mathrm{~d} W_{a}(t)+\sigma_{12} \mathrm{~d} W_{\theta}(t), \\
\mathrm{d} \theta=m_{\theta} \mathrm{dt}+\sigma_{21} \mathrm{~d} W_{a}(t)+\sigma_{22} \mathrm{~d} W_{\theta}(t) .
\end{array}\right.
$$

$m_{a}$ and $m_{\theta}$ are the drift coefficient. $\sigma_{11}, \sigma_{12}, \sigma_{21}, \sigma_{22}$ are the diffusion coefficient. $W_{a}(t)$ and $W_{\theta}(t)$ are the standard Winner process of independent of each other.

$$
\begin{aligned}
& m_{a}=\frac{b_{1}+b_{2}}{2} a+\frac{r_{V} V^{*}}{4 h_{E}^{4}} a^{3}+\frac{r_{V} E^{*} V^{*}}{2 h_{E}^{4}} a^{5}+\frac{5}{8}\left(\sigma_{1}^{2}+\sigma_{2}^{2}\right) a+\frac{1}{2 a} \sigma_{1}^{2} E^{* 2}+\frac{1}{2 a} \sigma_{2}^{2} V^{* 2}, \\
& b_{1}=r_{E}-\frac{2 r_{E} E^{*} V^{*}}{E_{0} h_{V}}-\frac{2 r_{E} h_{V} E^{*}}{E_{0} h_{V}}, b_{2}=r_{V}-\frac{2 r_{V} E^{* 4} V^{*}+2 r_{V} V^{*} h_{E}^{4}}{h_{E}^{4}}, m_{\theta}=0, \\
& \sigma_{11}^{2}=\frac{3}{8}\left(\sigma_{1}^{2}+\sigma_{2}^{2}\right) a^{2}+\frac{1}{2} \sigma_{1}^{2} E^{*}+\frac{1}{2} \sigma_{2}^{2} V^{*}, \sigma_{12}^{2}=\sigma_{21}^{2}=0, \sigma_{22}^{2}=\frac{1}{8}\left(\sigma_{1}^{2}+\sigma_{2}^{2}\right)+\frac{1}{2 a^{2}}\left(\sigma_{1}^{2} E^{* 2}+\sigma_{2}^{2} V^{* 2}\right) .
\end{aligned}
$$

Because of $\sigma_{12}^{2}=\sigma_{21}^{2}=0, a(t)$ is one dimensional Markov diffusion process.

$$
\mathrm{d} a=m_{a} \mathrm{dt}+\sigma_{11} \mathrm{~d} W_{a}(t) .
$$

FPK Method. Based on the Ito stochastic differential equation (5) and FPK method, we can see that the stochastic response diffusion process $p(t)$ satisfies the following FPK equation with initial conditions.

$$
\left\{\begin{array}{l}
\frac{\partial p}{\partial t}=-\frac{\partial\left[m_{a} p\right]}{\partial a}+\frac{1}{2} \frac{\partial^{2}\left[\sigma_{11}^{2} p\right]}{\partial a^{2}}, \\
p\left(a, t \mid a_{0}, t_{0}\right) \rightarrow \delta\left(a-a_{0}\right), t \rightarrow t_{0} .
\end{array}\right.
$$


$p\left(a, t \mid a_{0}, t_{0}\right)$ is the transferable probability density function of diffusion process $a(t)$. The Invariant measures of diffusion process $a(t)$ is steady-state probability density function $p_{s t}(a)$. The function $p_{s t}(a)$ is the solution of the following degenerate FPK equation.

$$
-\frac{\partial\left[m_{a} p\right]}{\partial a}+\frac{1}{2} \frac{\partial^{2}\left[\sigma_{11}^{2} p\right]}{\partial a^{2}}=0
$$

By calculating, we get the steady-state probability density function $p_{s t}(a)$ :

$$
p_{s t}(a)=c\left(1+c_{1} a^{2}\right)^{c_{2}}\left(\frac{1}{a^{2}}+c_{1}\right)^{c_{3}} e^{c_{4} a^{2}+c_{5} a^{4}}
$$

The coefficients of the function:

$$
\begin{aligned}
& c_{1}=\frac{3\left(\sigma_{2}^{2}+\sigma_{4}^{2}\right)}{4\left(\sigma_{2}^{2} E^{*}+\sigma_{4}^{2} V^{*}\right)}, c_{2}=\frac{4 b_{1}+4 b_{2}+5\left(\sigma_{2}^{2}+\sigma_{4}^{2}\right)}{3\left(\sigma_{2}^{2}+\sigma_{4}^{2}\right)}+\frac{\left(\sigma_{2}^{2} E^{*}+\sigma_{4}^{2} V^{*}\right) E^{* 2} V^{*}}{120\left(\sigma_{2}^{2}+\sigma_{4}^{2}\right)^{2}}-\frac{\left(\sigma_{2}^{2} E^{*}+\sigma_{4}^{2} V^{*}\right) V^{*}}{1080\left(\sigma_{2}^{2}+\sigma_{4}^{2}\right)^{3}}, \\
& c_{3}=\frac{2\left(\sigma_{2}^{2} E^{* 2}+\sigma_{4}^{2} V^{* 2}\right)}{\sigma_{2}^{2} E^{*}+\sigma_{4}^{2} V^{*}}, c_{4}=\frac{9 V^{*}\left(\sigma_{2}^{2} E^{*}+\sigma_{4}^{2} V^{*}\right)}{160\left(\sigma_{2}^{2}+\sigma_{4}^{2}\right)^{2}}-\frac{E^{* 2} V^{*}}{160\left(\sigma_{2}^{2}+\sigma_{4}^{2}\right)}, c_{5}=-\frac{V^{*}}{3840\left(\sigma_{2}^{2}+\sigma_{4}^{2}\right)}, \\
& b_{1}=r_{E}-\frac{2 r_{E} E^{*} V^{*}}{E_{0} h_{V}}-\frac{2 r_{E} h_{V} E^{*}}{E_{0} h_{V}}, b_{2}=r_{V}-\frac{2 E^{* 4} V^{*} r_{V}+2 V^{*} r_{V} h_{E}^{4}}{h_{E}^{4}} .
\end{aligned}
$$

The coefficient $c$ can be calculated by the normalization condition.

\section{The Stochastic Hopf Bifurcation Behavior}

When we study the problem of bifurcation of the nonlinear dynamic system, the invariant measure is an important characteristic value of the problem of the stochastic bifurcation [7]. Wang Bo and Qi Qianqian have used the Lyapunov stability theory and the numerical methods to get two bifurcation points of the model (1) where $E_{01}=5.153418$ and $E_{02}=7.288761$ about the control parameter $E_{0}$ [4]. We select the control parameter $E_{0}=7.0$ corresponding one linearly stable clear equilibrium states for the research. Corresponding equilibrium point: $E^{*}=1.377549$ and $V^{*}=0.816283$. Corresponding noise intensity values as shown in Table 1. Calculating the amplitude $a^{*}$ of the model (5) makes stable probability density function $p_{s t}(a)$ get the maximum value at $a^{*}$.

Table 1: Probabilities and positions of Hopf bifurcation occurrence of model (5)

\begin{tabular}{cccccccccc}
\hline condition & $\sigma_{1}$ & $\sigma_{2}$ & $a=a^{*}$ & $p_{s t}(a)$ & condition & $\sigma_{1}$ & $\sigma_{2}$ & $a=a^{*}$ & $p_{s t}(a)$ \\
\hline $\mathrm{A}$ & 0.1 & 0.1 & 0.4481 & 0.8905 & $\mathrm{E}$ & 0.1 & 0.1 & 0.4481 & 0.8095 \\
$\mathrm{~B}$ & 0.1 & 0.3 & 1.3809 & 0.7320 & $\mathrm{~F}$ & 0.3 & 0.1 & 1.8160 & 0.6806 \\
$\mathrm{C}$ & 0.1 & 0.5 & 3.9100 & 0.6378 & $\mathrm{G}$ & 0.5 & 0.1 & 4.0320 & 0.5164 \\
$\mathrm{D}$ & 0.1 & 0.8 & 5.9928 & 0.3249 & $\mathrm{H}$ & 0.8 & 0.1 & 6.0276 & 0.2649 \\
\hline
\end{tabular}




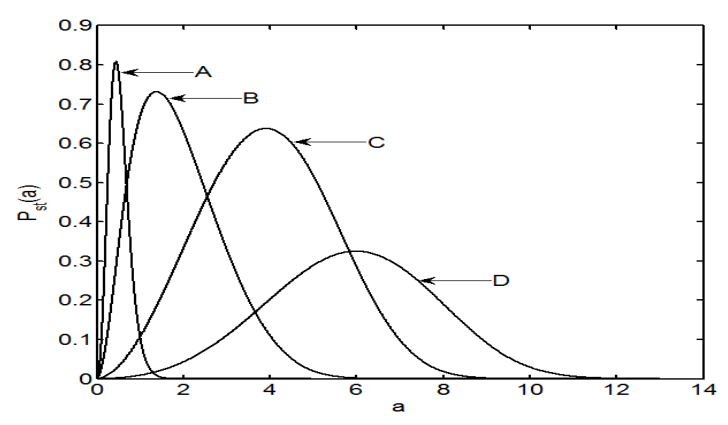

Fig. $2 p_{s t}(a)$ and position of stochastic Hopf bifurcation corresponding to noise intensity values A,B,C,D in Table 1 .

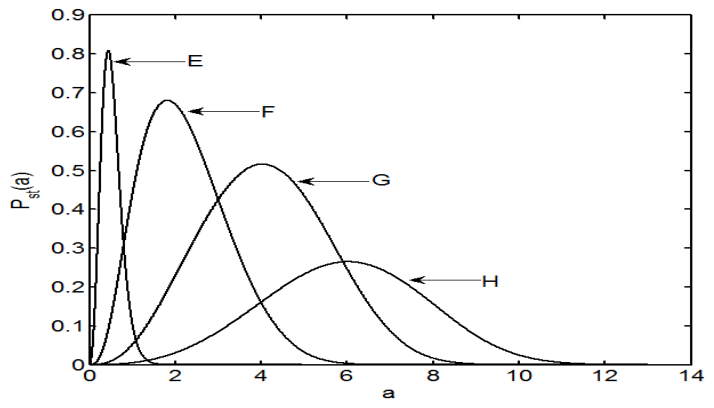

Fig. $3 p_{s t}(a)$ and position of stochastic Hopf bifurcation corresponding to noise intensity values E,F,G,H in Table 1.

From Fig. 2 and Fig. 3, we can see that the function $p_{s t}(a)$ get the minimum value at $a=0$. It shows that the stochastic model (2) is almost certainly unstable with stochastic excitation. At $a=a^{*}$, the function $p_{s t}(a)$ get the maximum value, the original stochastic system appear stochastic Hopf bifurcation. Appearing the location and probability of stochastic Hopf bifurcation as shown in table 1 . When the noise intensity is greater than zero, it could appear stochastic Hopf bifurcation with the probability meanings. The bifurcation location shifts with the increase of noise intensity. However, the probability density of appearing bifurcation is narrowing.

\section{Conclusions}

The bifurcation behavior of the stochastic system is different from the bifurcation behavior of the deterministic system. Due to the effect of stochastic factors is inevitable, studying the stochastic Hopf bifurcation of stochastic dynamical system is even more meaningful than studying the stability of the deterministic system at the equilibrium point. The stochastic Hopf bifurcation location shifts with the increase of noise intensity. If the bifurcation position reaches the threshold which appears turbid states, it is likely to lead the transitions between the different stable states. The shallow lake can change from clear equilibrium states to turbid equilibrium states.

\section{References:}

[1] B.E. Beisner, D.T. Haydon, K. Cuddington, Alternative stable states in ecology, Ecological Society of America. 1 (2003) 376-382.

[2] E.H. Vannes, M. Scheffer, Large species shifts triggered by small forces, American Naturalist. 164 (2004) 255-266.

[3] E.H. Vannes, M. Scheffer, Implications of spatial heterogeneity for catastrophic regime shifts in shallow lakes, Ecology. 86 (2005) 1797-1807.

[4] B. Wang, Q.Q. Qi, Application of the Conditional Nonlinear Optimal Perturbation Method in the shallow lake ecological degradation and restoration, Advances in meteorology. (2014).

[5] V.L. Oseledec, A multiplicative ergodic theorem Lyapunov characteristic numbers for dynamical systems, Translation of the Moscow Mathematics Society. 19 (1969) 197-231.

[6] R.Z. Khasminiskii, A limit theorem for solutions of differential equations with stochastic right hand sides, Theory of Probability and Applications. 11 (1966) 390-405.

[7] N.S. Namachivaya, Stochastic bifurcation, Applied Mathematics and Computation. 38 (1990) 101-159. 